\title{
Improvement by Iteration for Compact Operator Equations
}

\author{
By Ian H. Sloan
}

\begin{abstract}
The equation $y=f+K y$ is considered in a separable Hilbert space $H$, with $K$ assumed compact and linear. It is shown that every approximation to $y$ of the form $y_{1 n}=\Sigma^{n} a_{n i} u_{i}$ (where $\left\{u_{i}\right\}$ is a given complete set in $H$, and the $a_{n i}, 1 \leqslant i \leqslant n$, are arbitrary numbers) is less accurate than the best approximation of the form $y_{2 n}=f+$ $\Sigma^{n} b_{n i} K u_{i}$, if $n$ is sufficiently large. Specifically it is shown that if $y_{1 n}$ is chosen optimally (i.e. if the coefficients $a_{n i}$ are chosen to minimize $\left\|y-y_{1 n}\right\|$ ), and if $y_{2 n}$ is chosen to be the first iterate of $y_{1 n}$, i.e. $y_{2 n}=f+K y_{1 n}$, then $\left\|y-y_{2 n}\right\| \leqslant \alpha_{n}\left\|y-y_{1 n}\right\|$, with $\alpha_{n} \rightarrow 0$. A similar result is also obtained, provided the homogeneous equation $x=K x$ has no nontrivial solution, if instead $y_{1 n}$ is chosen to be the approximate solution by the Galerkin or Galerkin-Petrov method. A generalization of the first result to the approximate forms $y_{3 n}, y_{4 n}, \ldots$ obtained by further iteration is also shown to be valid, if the range of $K$ is dense in $H$.
\end{abstract}

1. Introduction. This paper is concerned with the approximate solution of the equation

$$
y=f+K y,
$$

where $y$ and $f$ belong to a separable Hilbert space $H$, and $K$ is a compact linear operator in $H$. The main results can also be extended to the eigenvalue equation $y=\lambda K y$, by setting $f=0$ and replacing $K$ by $\lambda K$. The whole discussion has immediate application to linear integral equations with square-integrable kernels, $H$ then being an $L^{2}$ space.

A common way of approaching Eq. (1) in practice is to seek an approximate solution of the form

$$
y_{1 n}=\sum_{i=1}^{n} a_{n i} u_{i}
$$

where $\left\{u_{i}\right\}$ is some suitable complete set of linearly independent elements of $H$, and the $a_{n i}$ are real or complex coefficients. Indeed, many numerical methods [2] , [4] for the approximate solution of integral equations (e.g. Galerkin method, collocation, least squares and variational methods) make use of an approximate solution of the form (2), differing from each other only in the choice of the coefficients.

The main purpose of the present paper is to show that the alternative form

$$
y_{2 n}=f+\sum_{i=1}^{n} b_{n i} K u_{i}
$$

Received April 21, 1975 ; revised December 30, 1975.

AMS (MOS) subject classifications (1970). Primary 45 L05, 45 B05, 65 R05; Secondary 45C05.

Key words and phrases. Integral equation, compact kernel, Galerkin method, Galerkin-Petrov method, eigenvalue problem. 
is always capable of better accuracy than (2), if $n$ is sufficiently large. Specifically, let $y_{1 n}^{*}$ and $y_{2 n}^{*}$ denote the best possible approximations of the forms (2) and (3), i.e. $y_{1 n}^{*}$ and $y_{2 n}^{*}$ are the approximations that minimize the respective Hilbert space error norms $\left\|y-y_{1 n}\right\|$ and $\left\|y-y_{2 n}\right\|$. Then it is shown in the following section (Theorem 1) that $\left\|y-y_{2 n}^{*}\right\| \leqslant \alpha_{n}\left\|y-y_{1 n}^{*}\right\|$, where $\alpha_{n} \rightarrow 0$. Indeed, it is shown that even the choice $y_{2 n}=f+K y_{1 n}^{*}$ (which is of the form (3), but not in general optimal) satisfies $\left\|y-y_{2 n}\right\| \leqslant \alpha_{n}\left\|y-y_{1 n}^{*}\right\|$, with $\alpha_{n} \rightarrow 0$. In other words, the best approximation of the form (2) is always improved by iteration (unless, of course, it is already exact), if $n$ is sufficiently large. An explicit expression for $\alpha_{n}$ is given in the theorem; hence information is available, in principle, on how large $n$ needs to be in any particular case for the improvement to be significant.

An extension of the result to the still more highly iterated approximate forms $y_{3 n}, y_{4 n}, \ldots$, given by

$$
y_{3 n}=f+K f+\sum_{i=1}^{n} c_{n i} K^{2} u_{i}, \text { etc. }
$$

is given in Theorem 2. The essential result is that the optimal approximation of the $m$ th kind $y_{m n}^{*}$ is always improved by a single iteration, if $n$ is sufficiently large, provided (for $m>1$ ) that the range of $K$ is dense in $H$, or what is equivalent, that the null space of $K^{*}$ is trivial.

For the various practical methods of the first kind, the solutions $y_{1 n}$ that arise are of course generally not optimal. For each such method, an interesting practical question is suggested by Theorem 1, namely: is it true that $y_{1 n}$ is necessarily improved by an iteration, if $n$ is sufficiently large? In the present work (Theorem 3 ) this question is answered in the affirmative for the Galerkin-Petrov method, thereby extending a result previously obtained [6], in the course of a study of degenerate-kernel methods for integral equations, for the ordinary Galerkin (or Bubnov-Galerkin) method.

The result for the Galerkin methods, that iteration necessarily improves the accuracy if $n$ is sufficiently large, is of practical importance, because the required iteration can be carried out without extra work, since the Galerkin methods already require the calculation of the quantities $K u_{i}, i=1, \ldots, n$. The fact that iteration of the Galerkin solution is sometimes beneficial has been observed previously [2], on the basis of practical experience. It is apparent from some recent calculations [7] that the benefit obtainable by the iteration can be very striking indeed, even in cases where $\|K\| \gg>1$.

2. Principal Result. Let $U_{n}$ be the subspace of $H$ spanned by $u_{1}, \ldots, u_{n}$, and let $P_{n}$ be the orthogonal projection operator onto $U_{n}$. Then $P_{n}$ has the properties $P_{n}^{2}=P_{n}=P_{n}^{*},\left\|P_{n}\right\|=1$. The sequence $\left\{u_{i}\right\}$ is assumed to be complete in $H$, hence $P_{n} x \rightarrow x$ for all $x \in H$.

The best approximation to $y$ of the form (2), i.e. the element $y_{1 n} \in U_{n}$ that minimizes $\left\|y-y_{1 n}\right\|$, is [1]

$$
y_{1 n}^{*}=P_{n} y
$$


Similarly, since $y-y_{2 n}$ can be written, with the aid of Eqs. (1) and (3), as

$$
y-y_{2 n}=K y-\sum_{i=1}^{n} b_{n i} K u_{i},
$$

it is seen that $\left\|y-y_{2 n}\right\|$ is minimized by choosing $\Sigma b_{n i} K u_{i}$ to be the orthogonal projection of $K y$ onto $K U_{n}$ (the span of $K u_{1}, \ldots, K u_{n}$ ). Thus, the best approximation to $y$ of the form (3) is

$$
y_{2 n}^{*}=f+P_{2 n} K y,
$$

where $P_{2 n}$ is the orthogonal projection operator onto $K U_{n}$.

THEOREM 1. If $y_{2 n}=f+K y_{1 n}^{*}$, then

$$
\left\|y-y_{2 n}^{*}\right\| \leqslant\left\|y-y_{2 n}\right\| \leqslant \alpha_{n}\left\|y-y_{1 n}^{*}\right\|,
$$

where

$$
\alpha_{n}=\left\|K-K P_{n}\right\| \rightarrow 0 .
$$

Proof. The first inequality follows from the optimal nature of $y_{2 n}^{*}$. To prove the second inequality, note that

$$
y-y_{2 n}=(f+K y)-\left(f+K y_{1 n}^{*}\right)=K\left(y-P_{n} y\right)=\left(K-K P_{n}\right)\left(y-P_{n} y\right),
$$

giving

$$
\left\|y-y_{2 n}\right\| \leqslant\left\|K-K P_{n}\right\|\left\|y-y_{1 n}^{*}\right\| .
$$

The proof is completed by the standard result $\left[5\right.$, p. 204] $\left\|K-K P_{n}\right\| \rightarrow 0$. (This follows, for example, by noting that

$$
\left\|K-K P_{n}\right\|=\left\|K^{*}-P_{n} K^{*}\right\|=\sup _{x \in H,\|x\|=1}\left\|K^{*} x-P_{n} K^{*} x\right\|
$$

hence if the contrary $\left\|K-K P_{n}\right\| \nrightarrow 0$ is supposed, then there exists $\epsilon>0$ and a sequence $\left\{x_{n}\right\} \subset H$ such that $\left\|K^{*} x_{n}-P_{n} K^{*} x_{n}\right\|>\epsilon,\left\|x_{n}\right\|=1$. Since $K^{*}$ is compact, $\left\{K^{*} x_{n}\right\}$ may be assumed to converge to an element $v \in H$, which implies

$$
\left\|K^{*} x_{n}-P_{n} K^{*} x_{n}\right\| \leqslant\left\|\left(I-P_{n}\right)\left(K^{*} x_{n}-v\right)\right\|+\left\|v-P_{n} v\right\| \rightarrow 0,
$$

a contradiction.) Q.E.D.

The key to the proof of Theorem 1 is the factorization carried out in Eq. (6), by exploiting the projection property $P_{n}^{2}=P_{n}$.

3. Generalization of Theorem 1. Let $y_{m n}$ be an expression of the form

$$
y_{m n}=\sum_{j=0}^{m-2} K^{j} f+\sum_{i=1}^{n} a_{m n i} K^{m-1} u_{i}, \quad m \geqslant 1
$$

where the $a_{m n i}$ are numbers; and let $P_{m n}$ denote the orthogonal projection operator onto $K^{m-1} U_{n}$ (the span of $K^{m-1} u_{1}, \ldots, K^{m-1} u_{n}$ ), so that $P_{m n}^{2}=P_{m n}=P_{m n}^{*}$ and $\left\|P_{m n}\right\|=1$. 
By repeated iteration of Eq. (1), the exact solution $y$ can be written in a form similar to (7), namely

$$
y=\sum_{j=0}^{m-2} K^{j} f+K^{m-1} y, \quad m \geqslant 1 .
$$

Hence, $y-y_{m n}$ can be written

$$
y-y_{m n}=K^{m-1} y-\sum_{i=1}^{n} a_{m n i} K^{m-1} u_{i}
$$

from which it follows that the approximation to $y$ of the form (7) that minimizes $\left\|y-y_{m n}\right\|$ is

$$
y_{m n}^{*}=\sum_{j=0}^{m-2} K^{j} f+P_{m n} K^{m-1} y .
$$

THEOREM 2. If $K^{*} x=0$ has no nontrivial solution in $H$, then

$$
\left\|y-y_{m+1, n}^{*}\right\| \leqslant\left\|y-\left(f+K y_{m n}^{*}\right)\right\| \leqslant \alpha_{m n}\left\|y-y_{m n}^{*}\right\|, \quad m \geqslant 1,
$$

where

$$
\alpha_{m n}=\left\|K-K P_{m n}\right\| \rightarrow 0 \text { as } n \rightarrow \infty
$$

Proof. It follows from Eq. (9) that

$$
f+K y_{m n}^{*}=\sum_{j=0}^{m-1} K^{j} f+K P_{m n} K^{m-1} y .
$$

Since this is of the form (7) with $m$ replaced by $m+1$, and since $y_{m+1, n}^{*}$ is the best possible approximation to $y$ of that form, the first inequality of the theorem is obvious. Furthermore, it follows from (10), and from (8) with $m$ replaced by $m+1$, that

$$
y-\left(f+K y_{m n}^{*}\right)=K^{m} y-K P_{m n} K^{m-1} y=\left(K-K P_{m n}\right)\left(K^{m-1} y-P_{m n} K^{m-1} y\right)
$$

hence

$$
=\left(K-K P_{m n}\right)\left(y-y_{m n}^{*}\right)
$$

$$
\left\|y-\left(f+K y_{m n}^{*}\right)\right\| \leqslant\left\|K-K P_{m n}\right\|\left\|y-y_{m n}^{*}\right\| .
$$

It only remains to prove that $\left\|K-K P_{m n}\right\| \rightarrow 0$ as $n \rightarrow \infty$. Since the null space of $K^{*}$ is trivial, it follows that the null space of $\left(K^{m}\right)^{*}=\left(K^{*}\right)^{m}$ is trivial; and hence that the set $\left\{K^{m} u_{1}, K^{m} u_{2}, \ldots\right\}$ is complete in $H$ (since otherwise there exists a nonzero element $x \in H$ satisfying $0=\left(x, K^{m} u_{i}\right)=\left(K^{m *} x, u_{i}\right)$ for each $i \geqslant 1$, which because $\left\{u_{i}\right\}$ is complete yields the contradiction $\left.K^{m *} x=0\right)$. Thus $P_{m n} x \rightarrow$ $x$ as $n \rightarrow \infty$ for each $x \in H$. The required result $\left\|K-K P_{m n}\right\| \rightarrow 0$ then follows exactly as for $\left\|K-K P_{n}\right\|$ in the previous section. Q.E.D.

Again the key step in the proof is a factorization, this time carried out in (11) and based on the property $P_{m n}^{2}=P_{m n}$.

4. Galerkin and Galerkin-Petrov methods. The simple Galerkin (or BubnovGalerkin) method [3, p. 223] makes use of an approximate solution of the form (2), with the coefficients being fixed by requiring the residual $y_{1 n}-f-K y_{1 n}$ to be orthogonal to $U_{n}$ (the span of $u_{1}, \ldots, u_{n}$ ), or equivalently, by requiring that 


$$
P_{n}\left(y_{1 n}-f-K y_{1 n}\right)=0
$$

where $P_{n}$ is the orthogonal projection operator onto $U_{n}$. Since $P_{n} y_{1 n}=y_{1 n}$, it follows that $y_{1 n}$ satisfies an equation of the second kind,

$$
y_{1 n}=P_{n} f+P_{n} K y_{1 n} .
$$

The more general Galerkin-Petrov method [3, p. 223] again makes use of an approximate solution of the form (2), but it uses a second set of linearly independent functions $\left\{v_{i}\right\}$ to determine the coefficients: Eq. (12) is replaced by

$$
Q_{n}\left(y_{1 n}-f-K y_{1 n}\right)=0,
$$

where $Q_{n}$ is the orthogonal projection operator onto $V_{n}$, the span of $v_{1}, \ldots, v_{n}$. Obviously, the Galerkin-Petrov method includes the ordinary Galerkin method as a special case.

Some restriction is necessary on the choice of $\left\{v_{i}\right\}$ in the Galerkin-Petrov method, to avoid the possibility of $Q_{n} y_{1 n}$ vanishing, or becoming very small. A suitable restriction [3, p. 224], assumed in the present work, is that the aperture $\theta\left(U_{n}, V_{n}\right)$ between $U_{n}$ and $V_{n}$, which is defined by [3, p. 205]

$$
\theta\left(U_{n}, V_{n}\right)=\left\|P_{n}-Q_{n}\right\|,
$$

should be strictly less than 1 in the limit,

$$
\varlimsup_{n \rightarrow \infty} \theta\left(U_{n}, V_{n}\right)<1 .
$$

It follows easily from this condition and from the completeness of the set $\left\{u_{i}\right\}$ that the set $\left\{v_{i}\right\}$ is necessarily complete.

The following theorem asserts that the approximate solution by the GalerkinPetrov method is necessarily improved by an iteration for $n$ sufficiently large, provided that 1 is not an eigenvalue of $K$.

THEOREM 3. If the homogeneous equation $x=K x$ has only the trivial solution, and if $y_{1 n}$ is the approximate solution of (1) by the Galerkin-Petrov method and if $y_{2 n}$ is defined by $y_{2 n}=f+K y_{1 n}$, then

$$
\left\|y-y_{2 n}\right\| \leqslant \beta_{n}\left\|y-y_{1 n}^{*}\right\| \leqslant \beta_{n}\left\|y-y_{1 n}\right\|,
$$

where $\beta_{n} \rightarrow 0$ as $n \rightarrow \infty$.

An explicit expression for $\beta_{n}$ is given by Eq. (26).

Proof. Since $K$ is compact and does not have 1 as an eigenvalue, the solution of Eq. (1) is unique and exists for any $f \in H$, and can be written

$$
y=(I-K)^{-1} f
$$

where $I$ is the identity operator, and $(I-K)^{-1}$ is a bounded linear operator in $H$.

The first step in the proof is to obtain from (14) an equation of the second kind for $y_{1 n}$, similar to the Galerkin equation (13). From the condition (15), it follows [3, p. 206] that for $n$ sufficiently large, say $n \geqslant n_{0}, Q_{n}$ maps $U_{n}$ one-one onto $V_{n}$. 
Thus, if $\bar{Q}_{n}$ denotes the operator $Q_{n}$ with its domain restricted to $U_{n}$, the inverse $\bar{Q}_{n}^{-1}$ exists for $n \geqslant n_{0}$. Furthermore, the inverses are uniformly bounded,

$$
\left\|\bar{Q}_{n}^{-1}\right\| \leqslant M, \quad n \geqslant n_{0} .
$$

By applying $\bar{Q}_{n}^{-1}$ to Eq. (14), and using $Q_{n} y_{1 n}=\bar{Q}_{n} y_{1 n}$, we obtain the desired equation

$$
y_{1 n}=\Pi_{n} f+\Pi_{n} K y_{1 n}, \quad n \geqslant n_{0},
$$

where

$$
\Pi_{n}=\bar{Q}_{n}^{-1} Q_{n}
$$

In particular, if $V_{n}=U_{n}$, then $\Pi_{n}=Q_{n}=P_{n}$, and (18) reduces to the Galerkin equation (13).

In the general case, it follows from (19) that the range of $\Pi_{n}$ is $U_{n}$, and that $\Pi_{n}^{2}=\Pi_{n}$, so that $\Pi_{n}$ is a projection operator (in general not orthogonal) onto $U_{n}$. From (17), $\left\|\Pi_{n}\right\|$ is uniformly bounded,

$$
\left\|\Pi_{n}\right\| \leqslant M, \quad n \geqslant n_{0} .
$$

It also follows from (19) that $\Pi_{n} P_{n}=P_{n}$ and that $Q_{n} \Pi_{n}=Q_{n}$; hence $I-\Pi_{n}$ can be written in either of the forms

$$
I-\Pi_{n}=\left(I-\Pi_{n}\right)\left(I-P_{n}\right)=\left(I-Q_{n}\right)\left(I-\Pi_{n}\right) .
$$

Since $y_{2 n}=f+K y_{1 n}$, where $y_{1 n}$ satisfies (18), it follows immediately that $y_{1 n}$ $=\Pi_{n} y_{2 n}$, hence $y_{2 n}$ satisfies

$$
y_{2 n}=f+K \Pi_{n} y_{2 n},
$$

an equation of the second kind with compact kernel $K \Pi_{n}$.

The operator $K \Pi_{n}$ converges in norm to $K$, because by virtue of (21) and (20),

$$
\left\|K-K \Pi_{n}\right\|=\left\|\left(K-K Q_{n}\right)\left(I-\Pi_{n}\right)\right\| \leqslant\left\|K-K Q_{n}\right\|(1+M),
$$

in which $\left\|K-K Q_{n}\right\| \rightarrow 0$ by the same argument as used for $\left\|K-K P_{n}\right\|$ in Section 2. It follows that $\left(I-K \Pi_{n}\right)^{-1}$ exists as a bounded linear operator for $n$ sufficiently large, and that it converges in norm to the bounded operator $(I-K)^{-1}$. The solution of (22) can therefore be written, for $n$ sufficiently large, as

$$
y_{2 n}=\left(I-K \Pi_{n}\right)^{-1} f_{\text {. }}
$$

It follows from (16) and (23) that

$$
\begin{aligned}
y-y_{2 n} & =\left[(I-K)^{-1}-\left(I-K \Pi_{n}\right)^{-1}\right] f=\left(I-K \Pi_{n}\right)^{-1}\left(K-K \Pi_{n}\right)(I-K)^{-1} f \\
& =\left(I-K \Pi_{n}\right)^{-1}\left(K-K \Pi_{n}\right) y .
\end{aligned}
$$

On using $\Pi_{n} P_{n}=P_{n}$, the last factor can be written as 


$$
\left(K-K \Pi_{n}\right) y=\left(K-K \Pi_{n}\right)\left(y-P_{n} y\right)
$$

hence we obtain

$$
\left\|y-y_{2 n}\right\| \leqslant\left\|\left(I-K \Pi_{n}\right)^{-1}\right\|\left\|K-K \Pi_{n}\right\|\left\|y-P_{n} y\right\|=\beta_{n}\left\|y-y_{1 n}^{*}\right\|
$$

where

$$
\beta_{n}=\left\|\left(I-K \Pi_{n}\right)^{-1}\right\|\left\|K-K \Pi_{n}\right\| .
$$

The limit $\beta_{n} \rightarrow 0$ follows from $\left\|\left(I-K \Pi_{n}\right)^{-1}\right\| \rightarrow\left\|(I-K)^{-1}\right\|$ and the result $\left\|K-K \Pi_{n}\right\| \rightarrow 0$ proved above.

The remaining inequality $\left\|y-y_{1 n}^{*}\right\| \leqslant\left\|y-y_{1 n}\right\|$ follows from the optimal property of $y_{1 n}^{*}$. Q.E.D.

The key to the rapid convergence established for the Galerkin-Petrov method is the factorization carried out in (24).

The bound on $\left\|y-y_{2 n}\right\|$ given by (25) may be very large if $K$ has an eigenvalue close to 1 , so that it may occasionally be useful to note that even in the worst case $\left\|y-y_{2 n}\right\|$ is also bounded by

$$
\left\|y-y_{2 n}\right\|=\left\|(f+K y)-\left(f+K y_{1 n}\right)\right\| \leqslant\|K\|\left\|y-y_{1 n}\right\| .
$$

However, it has been found in practical calculations [7] that the iterated Galerkin approximation retains a marked superiority over the Galerkin approximation itself, even in cases where there is an eigenvalue of $K$ very near to 1 .

5. Acknowledgements. I am indebted to Professor A. N. Mitra and the Department of Physics and Astrophysics at Delhi University for their hospitality at the time this research was carried out, and to the Leverhulme Foundation for the award of a fellowship.

Department of Applied Mathematics

University of New South Wales

Kensington, N.S.W. 2033, Australia

1. N. I. AHIEZER \& I. M. GLAZMAN, Theory of Linear Operators in Hilbert Space, vol. 1, GITTL, Moscow, 1950; English transl., Ungar, New York, 1961, Chapter 1. MR 13, 358; 41 \#9015a.

2. C. T. H. BAKER, "Expansion methods," Numerical Solution of Integral Equations, L. M. Delves and J. Walsh (Editors), Clarendon Press, Oxford, 1974, Chapter 7.

3. M. A. KRASNOSEL'SKIĬ, G. M. VAĬNIKKO, P. P. ZABREĬKO, Ja. B. RUTICKIĬ \& V. Ja. STECENKO, Approximate Solution of Operator Equations, "Nauka", Moscow, 1969; English transl., Wolters-Noordhoff, Groningen, 1972. MR 41 \#4271.

4. S. G. MIHLIN \& H. L. SMOLICKIĬ, Approximate Methods for Solution of Differential and Integral Equations, "Nauka", Moscow, 1965; English transl. by Scripta Technica, Modern Analytic and Computational Methods in Science and Mathematics, vol. 5, American Elsevier, New York, 1967, Chapter 4. MR 33 \#855; 36 \#1 108.

5. F. RIESZ \& B. SZ.-NAGY, Functional Analysis, Akad. Kiadó, Budapest, 1952, 2nd ed., 1953; English transl. of 2nd ed., Ungar, New York, 1955. MR 17, 175.

6. I. H. SLOAN, "Error analysis for a class of degenerate-kernel methods," Numer. Math., v. 25, 1976, pp. 231-238.

7. I. H. SLOAN, B. J. BURN \& N. DATYNER, "A new approach to the numerical solution of integral equations," J. Computational Phys., v. 18, 1975, pp. 92-105. 